\title{
Pre-existing arterial pathologic changes affecting arteriovenous fistula patency and cardiovascular mortality in hemodialysis patients
}

\author{
Ja Young Lee and Young Ok Kim
}

Department of Internal Medicine, College of Medicine, The Catholic University of Korea, Seoul, Korea

Received: August 2, 2017

Accepted: August 6, 2017

\section{Correspondence to}

Young Ok Kim, M.D.

Department of Internal

Medicine, College of Medicine,

Uijeongbu St. Mary's Hospital,

The Catholic University of Korea,

271 Cheonbo-ro, Uijeongbu 11765,

Korea

Tel: $+82-31-820-3347$

Fax: +82-31-820-2719

E-mail: cmckyo@catholic.ac.kr
The radiocephalic arteriovenous fistula (AVF) provides optimal vascular access for hemodialysis; it has a higher long-term patency rate and fewer complications than other vascular access methods. However, the AVF has a high primary failure rate. The presence of small-diameter vessels at anastomosis sites is an important risk factor for AVF failure. However, in a recent study, despite selecting an adequate artery and vein for creating an AVF by routine preoperative vascular mapping, AVF maturation and primary failure occurred. Thus, pre-existing arteriosclerosis at AVF anastomosis sites likely contributes to AVF failure. In this review, we discuss the relationship between pathologic changes and AVF patency in hemodialysis patients. Because arteriosclerosis of the major arteries such as the coronary and carotid arteries is associated with cardiovascular mortality, we also review the impact of arteriosclerosis of upper arm arteries at AVF anastomosis sites on cardiovascular mortality in hemodialysis patients.

Keywords: Renal dialysis; Arteriovenous fistula; Intimal hyperplasia; Intima-media thickness; Vascular calcification

\section{INTRODUCTION}

The radiocephalic arteriovenous fistula (AVF) is considered the best option for vascular access in hemodialysis (HD) patients. Disease Outcomes Quality Initiative guidelines recommend a fistula-first strategy when initiating HD [1]. This has resulted in a dramatic increase in the incidence of AVF, but the use of permanent catheters has increased and primary failure remains an issue [2-7]. Although preoperative vascular mapping was performed in a previous study to identify an artery and vein of adequate size for AVF creation, the primary failure rate did not decrease [8]. This suggests that in addition to old age, diabetes mellitus (DM), and a small-diameter vessel, pre-existing arteriosclerotic changes at AVF anastomosis sites may affect AVF maturation [8,9].
Cardiovascular disease is a major cause of morbidity and mortality in end-stage renal disease (ESRD) patients $[10,11]$. The cardiovascular mortality rate in ESRD patients adjusted for age, sex, and DM incidence is 10- to 20-fold higher than that in the general population [12]. Sarnak [13] explained the higher mortality rate in ESRD patients in terms of cardiac alterations in cardiac geometry, including left ventricular hypertrophy; atherosclerosis, including coronary artery disease; and arteriosclerosis. Arteriosclerosis occurs in large arteries such as the aorta and carotid arteries [13-15]. However, arteriosclerosis can develop in the radial artery, which is used for AVF. Therefore, pre-existing arteriosclerotic changes at AVF anastomosis sites could be related to cardiovascular mortality [16-20].

Two types of pathologic changes occur in arteries: atherosclerosis and arteriosclerosis. Atherosclerosis involves 
macrophage, lymphocyte, and lipid deposits in the coronary and carotid arteries [21,22]. Clinically eccentric luminal narrowing due to atherosclerosis causes cerebral and myocardial infarcts. Arteriosclerosis involves smooth muscle cell proliferation at the medial layer and movement into the intima (so-called intimal hyperplasia) $[16,17,23]$. Intimal hyperplasia is a common pathologic finding in venous stenosis, which is a common cause of vascular access failure. It can also be found in the radial artery [23]. Clinically concentric wall thickening due to intima-media thickening causes arterial stiffening, which is involved in cardiovascular mortality [24-26]. Vascular calcification can be found in both, but in different locations (the intimal layer in atherosclerosis and medial layer in intimal hyperplasia) [27]. Arteriosclerosis, which occurs in the radial artery of ESRD patients undergoing AVF, is mainly composed of intimal hyperplasia, intima-media thickening, and medial calcification $[9,16,17,19]$. This study evaluated the effect of pathologic changes in the arteries on AVF patency and cardiovascular mortality in ESRD patients.

\section{PATHOPHYSIOLOGY OF EARLY AVF FAILURE AND THE ROLE OF THE INFLOW ARTERY}

Thrombosis due to venous stenosis is the main cause of AVF failure. Venous stenosis is caused by neo-intimal hyperplasia $[28,29]$. After AVF creation, high pressure in the artery increases blood flow in the AVF, which induces venous wall thickening (arterialized vein). The increased venous diameter and blood flow reinforce arterial dilation, resulting in venous maturation. Wall shear stress is directly proportional to blood flow. Increased wall shear stress inhibits intimal hyperplasia, causing outward remodeling. In contrast, decreased wall shear stress due to decreased blood flow does not inhibit intimal hyperplasia or promote outward remodeling, resulting in luminal narrowing [30-32]. Therefore, pre-existing pathologic changes in the arteries before AVF creation cannot produce adequate blood flow for AVF maturation, resulting in early AVF failure [19,23].

\section{ACQUISITION OF ARTERIAL SPECIMENS}

In general, the opportunity to collect arterial specimens for the diagnosis of arterial disease is limited. So, studies of the pathologic findings of arterial disease are usually performed in coronary artery bypass graft (CABG) and organ transplant patients $[16,17,19]$. Obtaining an arterial specimen during AVF creation in ESRD patients is easy and safe, and it provides information on early pathologic changes. We reported that an arterial biopsy during AVF creation did not affect AVF patency [33]. However, it is not technically possible to obtain a whole circumferential artery sample as the ends of veins are connected to the sides of arteries.

\section{EFFECT OF ARTERIAL PATHOLOGIC CHANGES ON EARLY AVF FAILURE}

\section{Intimal hyperplasia}

Incidence of and risk factors for intimal hyperplasia of the radial artery in ESRD patients

Intimal hyperplasia is defined as the presence of cells and fibrous tissue between the endothelium and internal elastic lamina [17]. The radial and internal thoracic arteries are commonly used as conduits in CABG. However, because the radial artery has a higher incidence of intimal hyperplasia than the internal thoracic artery, the internal thoracic artery is now more commonly used [17]. The incidence of intimal hyperplasia of the radial artery varies. Allon et al. [34] reported that among 50 ESRD patients, none of the arteries obtained at the time of AVF creation had intimal hyperplasia. However, others reported values of $56 \%$ to $100 \%[16,23,35,36]$. It is unclear why the incidence of intimal hyperplasia varies among studies. The diagnostic criteria for intimal hyperplasia, degree of renal failure, age, race, and primary renal disease (e.g., DM) might play a role in the variable incidence of intimal hyperplasia. Chitalia et al. [9] reported that the intima thickness was increased in 10 brachial arteries and seven radial arteries of pre-dialysis and dialysis patients compared to controls. Johansson et al. $[17,19]$ used high-resolution ultrasound to examine the intimal and medial thicknesses in ESRD and healthy subjects. The intima and media in ESRD patients were $39 \%$ and $18 \%$, respectively, thicker than those in healthy subjects. The incidence of intima hyperplasia was $76.3 \%$ to $94 \%$ in patients with mild-to-moderate renal insuffi- 
ciency and coronary artery disease. Other risk factors for intimal hyperplasia are old age, DM, hypertension, and cardiovascular disease [23,37]. Because most ESRD patients have these risk factors, they are expected to show frequent intimal hyperplasia of the radial artery.

Effect of intimal hyperplasia of the radial artery on AVF patency There are only two studies on the relationship between AVF patency and intimal hyperplasia of the radial artery [21,34]. We evaluated the degree of intimal hyperplasia of the radial artery by histologic examination in 59 ESRD patients undergoing radiocephalic AVF creation [21]. The patients were followed for up to 1 year for AVF patency. AVF failure was defined as complete obstruction of the AVF or blood flow insufficient to support a dialysis blood flow rate of $\geq 200 \mathrm{~mL} / \mathrm{min}$. The AVF failure rate within 1 year in patients with intimal hyperplasia was higher than that in those without intimal hyperplasia. The intima in the failed AVF group was thicker than that in the patent group $(93.1 \pm 37.5 \mathrm{~mm}$ vs. $45.6 \pm 17.4$ $\mathrm{mm}, p<0.001)$. In contrast, Allon et al. [34] reported that AVF patency was not related to radial artery intimal hyperplasia. None of 50 patients had intimal hyperplasia. Another study [36] reported that the mean intima thickness of the radial artery was $12.5 \mathrm{~mm}$ in 73 ESRD patients undergoing an arteriovenous graft operation, which is thinner than in our work; the reason for this discrepancy is unknown. We suggest that demographic differences such as ethnicity, age, sex, and underlying disease, and differences in radial artery size led to the discrepant results in terms of the severity and frequency of intimal hyperplasia. In the above study [36], most of the patients were African-American, and patients with a minimum radial artery diameter of $2 \mathrm{~mm}$ were included. Our study included only Koreans, and venous mapping was not performed before the operations. The selection of vascular access was determined based on the surgeon's clinical experience. As a result, our study might have included smaller-diameter radial arteries. Chowdhury et al. [19] reported that the frequency of intimal hyperplasia was higher in small radial arteries. Therefore, the incidence and severity of intimal hyperplasia was higher and greater, respectively, in our work than in Allon's data [34,36]. Tripathi et al. [38] reported that the mean intima thickness of the radial artery was $49.9 \mathrm{~mm}$ in 32 Indian ESRD patients, which is similar to our result.
However, there was a limitation that the entire circumferential arterial wall could not be acquired during AVF. Intimal hyperplasia was evenly distributed throughout the wall of the radial artery in a study involving CABG, in which circumferential artery samples were obtained $[17,19]$. In addition to arterial intimal hyperplasia, pathologic changes in veins are important as venous stenosis is related to AVF patency. In conclusion, AVF patency is determined by the incidence and severity of pre-existing intimal hyperplasia of the radial artery.

\section{Intima-media thickness}

Intima-media thickness (IMT) is the sum of the thicknesses of the intimal and medial layers. Intimal hyperplasia starts with smooth cell proliferation in the medial layer and extends to the intimal layer, making it thicker $[16,17,23]$. The radial artery IMT of ESRD patients is thicker than that of healthy controls $[20,39,40]$ and can be easily identified by ultrasound sonography. We evaluated the accuracy of measurements of radial artery IMT using ultrasonography in 43 ESRD patients [39]. The value of the radial arterial IMT measured by sonographic examination correlated significantly with that by histological examination in 43 ESRD patients $(r=0.786, p<0.001)$. Johansson et al. [20] measured the intima, media, and IMT in ESRD patients and healthy controls by high-resolution ultrasonography; all were thicker in ESRD patients than in healthy controls, suggesting that the increased IMT in ESRD patients plays a role in cardiovascular events and AVF failure. We evaluated the impact of radial IMT on AVF failure in 90 ESRD patients for 1 year after the AVF operation [33]. The mean IMT was greater in the failed group $(n=31)$ than in the patent group $(\mathrm{n}=59 ; 486 \pm 130 \mu \mathrm{m}$ vs. $398 \pm 130 \mu \mathrm{m}$, $p=0.004)$. The AVF patency rate was lower in patients with an IMT $\geq 500 \mu \mathrm{m}(\mathrm{n}=26)$ than in patients with an $\mathrm{IMT}<500 \mu \mathrm{m}(\mathrm{n}=64 ; p=0.017)$ (Fig. 1). The mechanism underlying the effect of increased IMT on AVF failure is similar to that of intimal hyperplasia. We suggest that an increased IMT leads to insufficient arterial blood flow for AVF maturation. Other than ours, no study has evaluated the effect of radial artery IMT on AVF patency; thus, further work is required. 


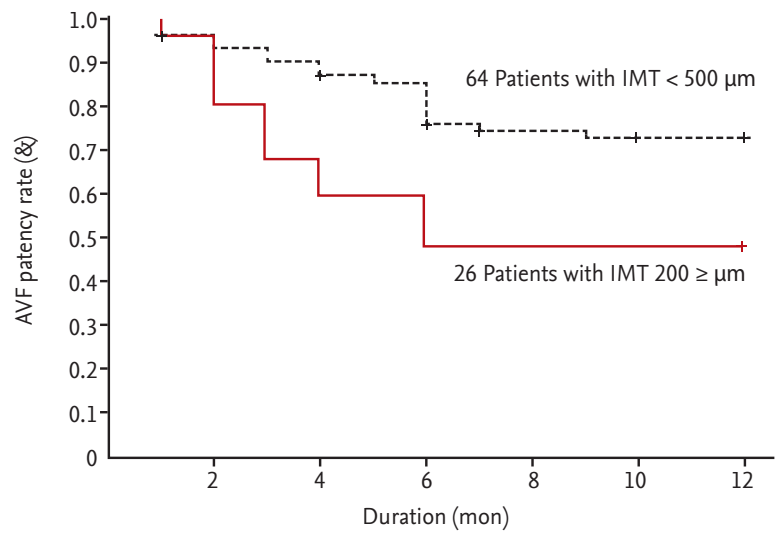

Figure 1. Arteriovenous fistula (AVF) patency rate in patients with an intima-media thickness (IMT) $\geq 500 \mu \mathrm{m}(\mathrm{n}=26)$ or $<$ $500 \mu \mathrm{m}(\mathrm{n}=64)$. Five patients who died with a patent AVF before the study end point were included as censored data. $p=$ 0.017 vs. the patent group by the Kaplan-Meier method and log-rank test. Adapted from Kim et al. [33].

\section{Arterial micro-calcification}

Pathogenesis and arterial micro-calcification type in ESRD patients

Several factors play a role in arterial micro-calcification (AMiC) in ESRD patients. Hypercalcemia, hyperphosphatemia, dyslipidemia, hypertension, DM, and uremic toxin induce vascular calcification $[41,42]$. This leads to osteoblastic conversion of vascular smooth muscle cells [43]. The process is facilitated by decreased levels of inhibitors of vascular calcification such as fetuin A, matrix Gla protein, osteopontin, osteoprotegerin, and pyrophosphate. Along with uremic toxin, oxidative stress and inflammation, which are commonly found in ESRD patients, are involved in vascular calcification [41,42]. Shroff et al. [44] demonstrated that dialysis accelerates AMiC by triggering smooth muscle cell apoptosis. AMiCs are found in different layers depending on the artery in question. Intimal calcification occurs in atherosclerosis, which is associated with atherosclerotic plaques and lipid accumulation. Clinically, intimal calcification results in stenotic lesions such as ischemic heart disease and cerebrovascular accidents $[41,42]$. In contrast, medial calcification is commonly observed in arteriosclerosis. It increases arterial stiffening, leading to left ventricular hypertrophy and congestive heart failure $[41,42]$. AMiC in the radial artery of ESRD patients is usually found in the medial area and can be detected by von Kossa stain- ing. The incidence of AMiC in the radial or brachial artery is $36 \%$ to $54.5 \%$ [45-49].

\section{The impact of AMiC on AVF patency}

Three studies have evaluated the effect of AMiC on AVF patency. We performed von Kossa staining to detect pre-existing AMiC in ESRD patients undergoing AVF, and followed up AVF failure for 1 year [47]. Preoperative vascular mapping was not performed. Of the patients, 65.8\% had DM and 80.7\% $(\mathrm{n}=75)$ had a radiocephalic $\operatorname{AVF}(\mathrm{n}=92)$. Of 114 patients, $\mathrm{AMiC}$ was found in $37.7 \%$. Patients with AMiC ( $\mathrm{n}=43$ ) showed a higher AVF failure rate than patients without AMiC $(\mathrm{n}=71)$. Allon et al. [34] reported that the incidence of AMiC $(n=47)$ was not related to maturation failure in 50 ESRD patients (79\% vs. $68 \%, p=0.6)$. However, a higher maturation failure rate was related to a high $\mathrm{AMiC}$ score, which represents calcification severity $(1.3 \pm 0.8$ vs. $0.9 \pm 0.8, p=0.08)$. In another study, Allon et al. [48] assessed the relationship between AMiC and AVF patency in 127 ESRD patients. They performed preoperative vascular mapping, and the study population comprised $46.6 \%(n=59)$ patients with DM and $34.6 \%(n=44)$ with a radiocephalic AVF, which are lower proportions than in our study. The incidence of AMiC was 40\%, which is similar to our result. In contrast to our findings, AMiC was not correlated with non-maturation and unassisted primary failure. Allon et al. [36] data showed the opposite results from our findings, possibly for the same reason as the intimal hyperplasia results. Besides the difference in ethnicity, our study included more DM patients and more patients with a radiocephalic AVF, and we did not perform preoperative vascular mapping, relying instead on the surgeon's clinical experience to select the vascular access operation site. Consequently, more calcified radial arteries would be included in our study. Georgiadis et al. [50] assessed the effect of radial artery gross calcification on primary and secondary AVF patency rates by plain $\mathrm{X}$-ray in DM patients before radiocephalic AVF. Patients with gross calcification $(\mathrm{n}=39)$ showed lower primary and secondary AVF patency rates than those without gross calcification $(\mathrm{n}=33)$. This suggests that the severity of pre-existing gross calcification affects radiocephalic AVF patency. Further study is expected in the future. 


\section{EFFECT OF ARTERIAL PATHOLOGIC CHANG- ES ON CARDIOVASCULAR MORBIDITY AND MORTALITY}

\section{Intimal hyperplasia and IMT}

The high cardiovascular mortality rate in ESRD patients is due to the high incidence of left ventricular hypertrophy, atherosclerosis, and arteriosclerosis [13,51]. Intimal hyperplasia, an increased IMT, and AMiC, which are common pathologic changes, lead to arteriosclerosis of the radial artery $[23,33,42]$. These pathologic changes occur more frequently in ESRD patients than in the general population and exhibit a clustered distribution $[18,19,39]$. In contrast to atherosclerosis, which is related to luminal narrowing and obstruction, arteriosclerotic changes cause arterial stiffening, endothelial dysfunction (leading to hypertension), left ventricular hypertrophy, and congestive heart failure, which are the main causes of cardiovascular mortality [24-26]. Ruengsakulrach et al. [17] reported that among patients with severe coronary artery disease awaiting CABG in whom the radial artery was used as the conduit artery, $94 \%$ had intimal hyperplasia. This suggests that intimal hyperplasia of the radial artery is closely related to coronary artery disease. Di Vito et al. [52] studied the association between thin cap-fibro atheroma of the coronary arteries, which is at high risk of rupture, and the intima-media ratio of the radial artery (maximal intima thickness/ medial thickness, which is used as the intima hyperplasia index) in patients with symptomatic coronary artery

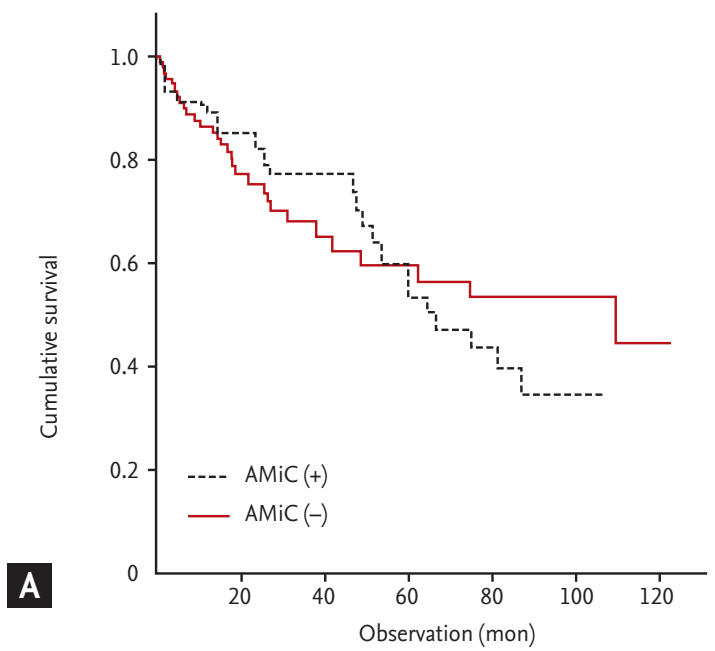

disease undergoing coronary procedures via the transradial route by frequency optical coherence tomography. The intima-media ratio of the radial artery was an independent risk factor for thin cap-fibroatheroma of the coronary arteries. MacKay et al. [18] examined the radial artery IMT in 25 patients with coronary artery disease and 20 healthy controls by Doppler ultrasound. Patients with coronary artery disease showed a significantly thickened IMT compared to healthy controls. Moreover, coronary artery disease was an independent risk factor for an increased IMT in the radial artery, irrespective of hypertension.

The effect of radial artery intimal hyperplasia or IMT on cardiovascular morbidity or mortality in ESRD patients has been reported. We studied the relationship between radial artery intimal hyperplasia and coronary artery disease in 56 ESRD patients undergoing their first AVF creation [23]. ESRD patients with radial artery intimal hyperplasia $(n=45)$ showed a higher incidence of coronary artery disease than those without radial artery intimal hyperplasia $(n=14 ; 48.5 \%$ vs. $14.2 \%, p=0.035)$. That study did not report cardiovascular mortality. Therefore, further research is required.

\section{AMiC}

Gross calcification of major arteries such as the aortic arch and coronary arteries is an independent risk factor for cardiovascular morbidity or mortality in ESRD patients as well as the general population [53]. However, only two studies have assessed the relationship of AMiC

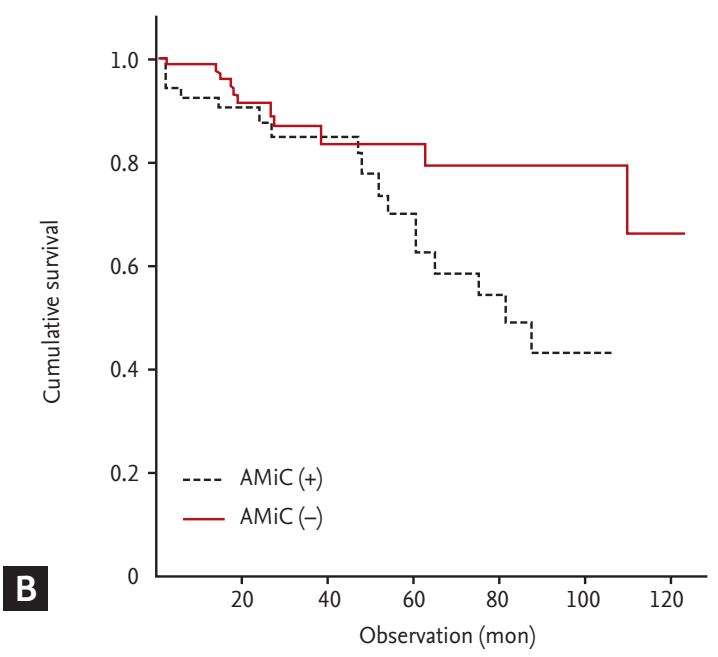

Figure 2. Kaplan-Meier survival curves for all-cause $(A: \log -r a n k=0.18, p=0.676)$ and cardiovascular mortality $(B: \log -$ rank $=$ $4.83, p=0.028)$. Adpated from Allon et al., with permission from John Wiley and Sons [46]. 
of the radial artery, confirmed histologically earlier than gross calcification by radiologic imaging, with cardiovascular mortality and morbidity. We evaluated the correlation of AMiC and aortic arch calcification by chest X-ray, arterial stiffness by pulse-wave velocity, and endothelial dysfunction by flow-mediated dilation in 65 ESRD patients undergoing AVF operations [45]. From the 65 cases, we obtained 53 radial artery specimens. AMiC had a proportional relationship with aortic calcification and arterial stiffness. However, endothelial dysfunction was positive in most of the patients, irrespective of AMiC. We investigated the effect of AMiC of the radial and brachial arteries on cardiovascular and all-cause mortality in 149 ESRD patients undergoing vascular access operations [46]; 125 radial artery specimens were obtained. AMiC was associated with cardiovascular mortality, but not all-cause mortality (Fig. 2). Janda et al. [54] reported that carotid artery IMT was predictive of radial artery AMiC in ESRD patients. The carotid artery IMT, which is considered a risk marker for atherosclerosis, is an independent risk factor for cardiovascular mortality in ESRD patients as well as in the general population. Therefore, AMiC increases cardiovascular mortality by inducing arteriosclerotic and atherosclerotic changes, including coronary artery disease, in ESRD patients.

\section{CONCLUSIONS}

Because radiocephalic AVF has a lower complication rate and longer patency, the radial artery is frequently used for vascular access. However, arteriosclerotic changes such as intimal hyperplasia, an increased IMT, and medial AMiC are often found in the radial artery of ESRD patients. This pathologic change can contribute to AVF failure and cardiovascular mortality.

\section{Conflict of interest}

No potential conflict of interest relevant to this article was reported.

\section{REFERENCES}

1. III. NKF-K/DOQI clinical practice guidelines for vascular access: update 2000. Am J Kidney Dis 2001;37(1 Suppl
1):S137-S181.

2. Lee J, Kim YS, Yoon SA, et al. Retrospective review of angiography before cannulation of newly created vascular accesses in hemodialysis patients. J Vasc Interv Radiol 2013;24:1309-1315.

3. Lok CE. Fistula first initiative: advantages and pitfalls. Clin J Am Soc Nephrol 2007;2:1043-1053.

4. Patel ST, Hughes J, Mills JL Sr. Failure of arteriovenous fistula maturation: an unintended consequence of exceeding dialysis outcome quality Initiative guidelines for hemodialysis access. J Vasc Surg 2003;38:439-445.

5. Lok CE, Sontrop JM, Tomlinson G, et al. Cumulative patency of contemporary fistulas versus grafts (2000-2010). Clin J Am Soc Nephrol 2013;8:810-818.

6. Combe C, Berard X. Dialysis: 'catheter last' not 'fistula first' in elderly patients. Nat Rev Nephrol 2013;9:632-634.

7. DeSilva RN, Patibandla BK, Vin Y, et al. Fistula first is not always the best strategy for the elderly. J Am Soc Nephrol 2013;24:1297-1304.

8. Peterson WJ, Barker J, Allon M. Disparities in fistula maturation persist despite preoperative vascular mapping. Clin J Am Soc Nephrol 2008;3:437-441.

9. Chitalia N, Ross L, Krishnamoorthy M, et al. Neointimal hyperplasia and calcification in medium sized arteries in adult patients with chronic kidney disease. Semin Dial 2015;28:E35-E40.

10. Di Lullo L, House A, Gorini A, Santoboni A, Russo D, Ronco C. Chronic kidney disease and cardiovascular complications. Heart Fail Rev 2015;20:259-272.

11. Schiffrin EL, Lipman ML, Mann JF. Chronic kidney disease: effects on the cardiovascular system. Circulation 2007;116:85-97.

12. Foley RN, Parfrey PS, Sarnak MJ. Clinical epidemiology of cardiovascular disease in chronic renal disease. Am J Kidney Dis 1998;32(5 Suppl 3):S112-S119.

13. Sarnak MJ. Cardiovascular complications in chronic kidney disease. Am J Kidney Dis 2003;41(5 Suppl):11-17.

14. London GM, Marchais SJ, Guerin AP, Metivier F, Adda H. Arterial structure and function in end-stage renal disease. Nephrol Dial Transplant 2002;17:1713-1724.

15. Klassen PS, Lowrie EG, Reddan DN, et al. Association between pulse pressure and mortality in patients undergoing maintenance hemodialysis. JAMA 2002;287:1548-1555.

16. Ejerblad S, Ericsson JL, Eriksson I. Arterial lesions of the radial artery in uraemic patients. Acta Chir Scand 1979;145:415-428. 
17. Ruengsakulrach P, Sinclair R, Komeda M, Raman J, Gordon I, Buxton B. Comparative histopathology of radial artery versus internal thoracic artery and risk factors for development of intimal hyperplasia and atherosclerosis. Circulation 1999;100:II139-II144.

18. MacKay AJ, Hamilton CA, McArthur K, et al. Radial artery hypertrophy occurs in coronary atherosclerosis and is independent of blood pressure. Clin Sci (Lond) 2001;100:509-516.

19. Chowdhury UK, Airan B, Mishra PK, et al. Histopathology and morphometry of radial artery conduits: basic study and clinical application. Ann Thorac Surg 2004;78:16141621.

20. Johansson M, Myredal A, Friberg P, Gan LM. High-resolution ultrasound showing increased intima and media thickness of the radial artery in patients with end-stage renal disease. Atherosclerosis 2010;211:159-163.

21. Stary HC, Chandler AB, Dinsmore RE, et al. A definition of advanced types of atherosclerotic lesions and a histological classification of atherosclerosis: a report from the Committee on Vascular Lesions of the Council on Arteriosclerosis, American Heart Association. Circulation 1995;92:1355-1374.

22. Ross R. The pathogenesis of atherosclerosis: a perspective for the 1990s. Nature 1993;362:801-809.

23. Kim YO, Song HC, Yoon SA, et al. Preexisting intimal hyperplasia of radial artery is associated with early failure of radiocephalic arteriovenous fistula in hemodialysis patients. Am J Kidney Dis 2003;41:422-428.

24. Sato M, Ogawa T, Sugimoto H, Otsuka K, Nitta K. Relation of carotid intima-media thickness and silent cerebral infarction to cardiovascular events and all-cause mortality in chronic hemodialysis patients. Intern Med 2012;51:2111-2117.

25. Szeto CC, Chow KM, Woo KS, et al. Carotid intima media thickness predicts cardiovascular diseases in Chinese predialysis patients with chronic kidney disease. J Am Soc Nephrol 2007;18:1966-1972.

26. Nishizawa Y, Shoji T, Maekawa K, et al. Intima-media thickness of carotid artery predicts cardiovascular mortality in hemodialysis patients. Am J Kidney Dis 2003;41(3 Suppl 1):S76-S79.

27. Amann K. Media calcification and intima calcification are distinct entities in chronic kidney disease. Clin J Am Soc Nephrol 2008;3:1599-1605.

28. Himmelfarb J, Saad T. Hemodialysis vascular access: emerging concepts. Curr Opin Nephrol Hypertens 1996;5:485-491.

29. Mousa AY, Dearing DD, Aburahma AF. Radiocephalic fistula: review and update. Ann Vasc Surg 2013;27:370-378.

30. Browne LD, Bashar K, Griffin P, Kavanagh EG, Walsh SR, Walsh MT. The role of shear stress in arteriovenous fistula maturation and failure: a systematic review. PLoS One 2015;10:e0145795.

31. Sivanesan S, How TV, Bakran A. Characterizing flow distributions in AV fistulae for haemodialysis access. Nephrol Dial Transplant 1998;13:3108-3110.

32. Ene-Iordache B, Mosconi L, Antiga L, et al. Radial artery remodeling in response to shear stress increase within arteriovenous fistula for hemodialysis access. Endothelium 2003;10:95-102.

33. Kim YO, Choi YJ, Kim JI, et al. The impact of intima-media thickness of radial artery on early failure of radiocephalic arteriovenous fistula in hemodialysis patients. J Korean Med Sci 2006;21:284-289.

34. Allon M, Litovsky S, Young CJ, et al. Medial fibrosis, vascular calcification, intimal hyperplasia, and arteriovenous fistula maturation. Am J Kidney Dis 2011;58:437-443.

35. Yan TK, Lin S, Jia JY, Li YP, Shang WY, Wei L. The histological changes in radial artery in uremia and their effects on arterial stiffness. Zhonghua Nei Ke Za Zhi 2010;49:577581.

36. Allon M, Litovsky S, Young CJ, et al. Correlation of pre-existing vascular pathology with arteriovenous graft outcomes in hemodialysis patients. Am J Kidney Dis 2013;62:1122-1129.

37. Kaufer E, Factor SM, Frame R, Brodman RF. Pathology of the radial and internal thoracic arteries used as coronary artery bypass grafts. Ann Thorac Surg 1997;63:1118-1122.

38. Tripathi V, Bansal S, Alok S, Ravi B, Devra AK, Saxena S. Histopathological changes of radial artery wall in patients of chronic kidney disease stage 5 undergoing AV fistula formation and their correlation with serum iPTH levels. Saudi J Kidney Dis Transpl 2015;26:884-889.

39. Ku YM, Kim YO, Kim JI, et al. Ultrasonographic measurement of intima-media thickness of radial artery in pre-dialysis uraemic patients: comparison with histological examination. Nephrol Dial Transplant 2006;21:715-720.

40. Simon A, Gariepy J, Chironi G, Megnien JL, Levenson J. Intima-media thickness: a new tool for diagnosis and treatment of cardiovascular risk. J Hypertens 2002;20:159169. 
41. Mizobuchi M, Towler D, Slatopolsky E. Vascular calcification: the killer of patients with chronic kidney disease. J Am Soc Nephrol 2009;20:1453-1464.

42. Paloian NJ, Giachelli CM. A current understanding of vascular calcification in CKD. Am J Physiol Renal Physiol 2014;307:F891-F900.

43. Yun YS, Yun SS, Kim HK, Kim YO. The histopathologic analysis of microcalcification of the radial artery in patients with end stage renal disease [abstract]. J Vasc Access 2011;12:173.

44. Shroff RC, McNair R, Figg N, et al. Dialysis accelerates medial vascular calcification in part by triggering smooth muscle cell apoptosis. Circulation 2008;118:1748-1757.

45. Kim HG, Park SC, Lee SL, et al. Arterial micro-calcification of vascular access is associated with aortic arch calcification and arterial stiffness in hemodialysis patients. Semin Dial 2013;26:216-222.

46. Yun YS, Choi SJ, Lee JY, et al. Impact of arterial microcalcification of the vascular access on cardiovascular mortality in hemodialysis patients. Hemodial Int 2014;18:54-61.

47. Choi SJ, Yoon HE, Kim YS, et al. Pre-existing arterial micro-calcification predicts primary unassisted arteriovenous fistula failure in incident hemodialysis patients. Semin Dial 2015;28:665-669.

48. Allon M, Robbin ML, Umphrey HR, et al. Preoperative arterial microcalcification and clinical outcomes of arteriovenous fistulas for hemodialysis. Am J Kidney Dis 2015;66:84-90.
49. Wang N, Yang J, Yu X, et al. Radial artery calcification in end-stage renal disease patients is associated with deposition of osteopontin and diminished expression of alpha-smooth muscle actin. Nephrology (Carlton) 2008;13:367-375.

50. Georgiadis GS, Georgakarakos EI, Antoniou GA, et al. Correlation of pre-existing radial artery macrocalcifications with late patency of primary radiocephalic fistulas in diabetic hemodialysis patients. J Vasc Surg 2014;60:462470.

51. Kim MK, Kim B, Lee JY, et al. Tissue Doppler-derived E/ e' ratio as a parameter for assessing diastolic heart failure and as a predictor of mortality in patients with chronic kidney disease. Korean J Intern Med 2013;28:35-44.

52. Di Vito L, Porto I, Burzotta F, et al. Radial artery intima-media ratio predicts presence of coronary thin-cap fibroatheroma: a frequency domain-optical coherence tomography study. Int J Cardiol 2013;168:1917-1922.

53. London GM, Guerin AP, Marchais SJ, Metivier F, Pannier B, Adda H. Arterial media calcification in end-stage renal disease: impact on all-cause and cardiovascular mortality. Nephrol Dial Transplant 2003;18:1731-1740.

54. Janda K, Krzanowski M, Gajda M, et al. Cardiovascular risk in chronic kidney disease patients: intima-media thickness predicts the incidence and severity of histologically assessed medial calcification in radial arteries. BMC Nephrol 2015;16:78. 